\title{
A Comprehensive Scoping Review Protocol of Using Living Labs to Explore Needs and Solutions for Older Adults with Dementia
}

This article was published in the following Dove Press journal: Smart Homecare Technology and TeleHealth

\author{
Henk Verloo (D) ${ }^{1,2}$ \\ Adrien Lorette (iD) ${ }^{2}$ \\ Carla Gomes da Rocha' \\ Joëlle Rosselet Amoussou (iD ${ }^{3}$ \\ Estelle Gillès de Pélichy ${ }^{2,4}$ \\ Alcina Matos Queiros ${ }^{5}$ \\ Montserrat Mendez Rubio ${ }^{2}$ \\ Armin von Gunten ${ }^{2}$ \\ 'School of Health Sciences, HES-SO \\ Valais / Wallis and Department of \\ Nursing Sciences, University of Applied \\ Sciences and Arts Western Switzerland, \\ Sion, CH-1950, Switzerland; \\ ${ }^{2}$ Department of Psychiatry, Service of \\ Old Age Psychiatry, Lausanne University, \\ Prilly-Lausanne, $\mathrm{CH}-1008$, Switzerland; \\ ${ }^{3}$ Psychiatry Library, Education and \\ Research Department, Lausanne \\ University Hospital and University of \\ Lausanne, Site of Cery, $\mathrm{CH}-\mathrm{I008}$, \\ Switzerland; ${ }^{4}$ Psychiatric Sector West, \\ Prangins Hospital, Prangins $\mathrm{CH}-\mathrm{I} 197$, \\ Switzerland; ${ }^{5}$ Geriatrics and Old Age \\ Psychiatry, Health and Social Services \\ Department, Directorate-General for \\ Social Cohesion Bâtiment Administratif \\ de la Pontaise, Lausanne $\mathrm{CH}-\mathrm{IOI} 4$, \\ Switzerland
}

Background: A significant number of living labs (LLs) have been developed across Europe and beyond. A fraction of those LLs have established a new approach to maintaining and studying the health, autonomy, and well-being of older adults with dementia. LLs interact with a broad set of stakeholders, including students, academic institutions, private companies, healthcare organizations, and patient representative bodies - even with other LLs. It is crucial to identify what kinds of co-creations should be done and how they can be facilitated through LLs. Despite a growing body of literature, a clear overview and understanding of the services, research, and clinical activities developed in different LL settings for older adults with dementia are still lacking.

Aim: The aim is to scope publications examining all types of LL activities which explore the needs of and suggest solutions for older adults with dementia, whether they live in the community or long-term healthcare facilities.

Methods: The bibliographic databases to be searched will include Embase.com, Medline Ovid SP, Pubmed (not medline[sb]) and Web of Science, without language or date restrictions. We will examine the bibliographies of all relevant articles found, conduct a search for unpublished studies, and perform a handsearch in relevant journals associated with LLs involved in healthcare (Electronic Journal for Virtual Organization and Networks, Technology Innovation Management Review, Journal of Engineering and Technology Management). We will consider publications in English, French, and German.

Results: Bibliographic database searches will be completed in March 2020, retrieved articles will be screened, and the entire study is expected to be completed by December 2020 .

Discussion: This comprehensive scoping review will provide a global indication of the types and extent of LL activities aimed at older adults with dementia, whether they live in the community or in long-term care facilities.

Keywords: living lab, primary healthcare, older adults, long-term facilities, co-creation, needs assessment, solutions, technology

\section{Introduction}

The world's population of people aged over 65 years old is growing rapidly; in Europe, their proportion will rise from $14 \%$ in 2010 to $28 \%$ in $2020 .{ }^{1}$ In Switzerland, female life expectancy is over 84 years old and male life expectancy is $82 .^{2}$ A significant fraction of these older adults will present with chronic diseases and will be particularly at risk of progressive psychopathological decline. ${ }^{3,4}$ According to the World Health Organisation, about $20 \%$ of people aged 65 years old or more have
Correspondence: Henk Verloo School of Health Sciences, HES-So Valais and Department of Nursing Sciences,

University of Applied Sciences and Arts

Western Switzerland, 5, Chemin de

l'Agasse, Sion CH-1950, Switzerland

Tel +4l 787698990

Email henk.verloo@hevs.ch

Smart Homecare Technology and TeleHealth 2020:7 19-27 
difficulties in carrying out some activities of daily living or instrumental activities of daily living, often due to reduced mobility, weakened muscular strength, and disorders linked to psychopathological problems. ${ }^{5}$ Innovative technologies or services are being used ever more frequently to provide fresh responses to health problems, particularly for those suffering from dementia. ${ }^{6,7}$ In parallel, more and more healthcare professionals and individual citizens want to participate in these innovations and voice their opinions on the definition of pertinent, innovative, and implementable solutions. ${ }^{8}$ Indeed, the field of health care is actively looking for open-innovation approaches to meeting the day to day complexities of clinical practice. One means of doing this is by driving collaborative multi-stakeholder inquiries that can better identify and respond to the complex health needs of community-dwelling older adults with cognitive impairment due to varied etiologies. With these ends in mind, we will explore open innovation in the health arena, suggesting that it has become a significant sector in pushing the limits of open methods and that this is challenging mainstream conceptions of the targets of health innovation. ${ }^{9}$ This context points to the utility to design methods oriented towards increasing the participation of users and stakeholders, whether non-specialists or professionals. ${ }^{10,11}$ The European Network of Living Labs (ENoLL) is an active promotor of the approach. ${ }^{12}$ LLs can turn the main beneficiary of the resolution of a problem or the major stakeholder in a phenomenon into an actor with a key role in a scientific process, ${ }^{13}$ and the innovators also get direct access to their target recipients. Furthermore, because LL solutions are developed under conditions which are closer to reality, they should reveal more effective solutions to their endusers' needs. ${ }^{12}$

\section{Living Labs}

Living Labs can be characterized in multiple ways and serve several purposes. They are both practice-driven organisations that facilitate and foster open, collaborative innovation, as well as real-life environments or arenas, where both open innovation and user innovation processes can be studied and experimented with, and where new solutions are developed. ${ }^{14}$

\section{Terminology}

The term Living Lab was first coined by Mitchell (2003) in the context of the community where the activities and interactions of ordinary home life could be observed, documented for later investigation, and experimentally manipulated. ${ }^{15}$
More specifically, LLs were seen as extended laboratory experiments whose objective was to gather more accurate, natural user information and data by observing long periods of day-to-day activities. This was considered by some authors as the American or original type of LL. ${ }^{16}$ American LL users are more rarely actively involved in the development of products or services: their roles are closer to those of being passive test subjects or research subjects. In comparison, European LLs are often short-term, small-scale, cocreation projects taking place in real-life environments and characterized by the following basic elements: a real-life setting, multiple stakeholder participation, a multi-methods approach, and co-creation. ${ }^{17}$ In 2006, the European LL movement attracted the attention of the European Union (EU) and the ENoLL was founded. ${ }^{18}$

\section{Definition}

There are many different definitions of a Living Lab depending on the domain (energy, technology, smart cities, etc.) and the author's field of research. However, the absence of a widely recognized definition ${ }^{19-21}$ indicates the lack of a common understanding of the concept and its underlying mechanisms and is also a symptom of their heterogeneity. ${ }^{22,23} \mathrm{~A}$ recent review by Leminen (2015) identified 70 different definitions of an LL. ${ }^{24}$ Despite the lack of a widely accepted definition, however, they commonly share the following two key elements: a real-life test and experimentation environment and stakeholders who are external to the research organization but co-involved in the innovation/research process. Schuurmans defined LL as "a multi-stakeholder organization set-up to carry out innovation projects that follow the principles of open and user innovation and focus on real-life experimentation." 25

Within the framework of our comprehensive scoping review, the definition presented by Bergvall-Kåreborn and Ståhlbröst seemed very suitable:

A Living Lab is a user-centric innovation milieu built on every-day practice and research, with an approach that facilitates user influence in open and distributed innovation processes engaging all relevant partners in real-life contexts, aiming to create sustainable values.

With regard to older adults with dementia in different healthcare settings, Bergvall-Kåreborn and Ståhlbröst also stated that an LL could be 
a pragmatic research environment, which openly engages all relevant partners with an emphasis on improving the real-life care of people living with dementia through the use of economically viable and sustainable innovation. ${ }^{23,26}$

\section{Key Components of a Living Lab}

LLs can be seen as settings for open innovation, which provide collaborative platforms for research, development, and experimentation in real-life contexts using particular methodologies and tools. ${ }^{27}$ LLs are driven by two principal philosophies: 1) involving users as co-creators of innovative outcomes on an equal basis with the other stakeholders, and 2) experimenting in real-world contexts. ${ }^{28}$ Følstad (2008) described nine characteristics of LLs, four of which they all display: discovery, evaluation, familiar contexts, and a focus on the medium-to-long term. The other five may or may not be displayed, thus contributing to the variety of LLs: investigation of the context, active roles for the users, technical testing, real-world contexts, and multiple settings. ${ }^{29}$ This illustrates how diverse LLs are in practice. By using LL platforms and methodologies, companies and healthcare institutions can reach beyond their own boundaries, follow an open-innovation model, ${ }^{30}$ and integrate outsiders into the co-creation of products, ${ }^{31}$ experiences, designs, quality implementation strategies, and service development. ${ }^{30}$ As part of the co-creation process, LLs often act as intermediaries or innovation facilitators by providing structure and governance to the co-creation process. ${ }^{32,33}$ Key components of LLs include information and communication technology (ICT), management, stakeholders, research, and their methods of co-creation and product testing. ${ }^{26}$ The ICT and infrastructure component reflects the role, which new and existing ICT can play in facilitating new means of cooperating and cocreating innovations among stakeholders. Management represents the ownership, organization, and policy aspects of LLs. An LL can be managed by consultants, companies, or researchers. LL partners and users bring their own specific knowledge and expertise to the collective, helping to achieve boundary-spanning knowledge transfer. Research symbolizes the collective learning and thinking that takes place in the LL and which should result in contributions to both theory and practice. Technological research partners can also provide direct access to such fora as panels of older adult testers of new products, which can benefit the outcome of a technological innovation with regards to criteria like ease of use. $^{26}$

\section{Living Labs Exploring Needs and Seeking Solutions with Older Adults with Dementia}

Dementia is a progressive, disabling, chronic disease affecting $5 \%$ of all people above 65 years old and over $40 \%$ of people over 90 years old. ${ }^{34}$ Typical symptoms include impairments of memory, thought, perception, speech, and reasoning. Initial impairments in performing complex tasks ultimately lead to an inability to perform even the most basic functional activities such as bathing and eating. Furthermore, it is also common to observe alterations in personality, behavior, and psychological functioning, such as symptoms of depression, apathy, and responsive behavior. ${ }^{35,36}$ The current demographic transition suggests that we should expect growing numbers of older and very old adults and a consequent rise in the numbers of older adults with dementia. This will surely lead to longer waiting lists for places in protected housing, nursing homes, and other long-term healthcare facilities. However, most older adults with dementia will have to survive in their own place of living, and if this could be facilitated the need for residential care would substantially decrease. At the moment, the vast majority of older adults in long-term care do indeed have problems with cognitive functioning. ${ }^{37}$ Older adults with psychopathological impairments need a great deal of support and assistance, and this need increases as the disease progresses. ${ }^{38}$ In cases of severe dementia, help is needed 24 hrs a day. ${ }^{39}$ Nevertheless, most older people prefer to stay in their own homes for as long as possible, even if they risk falls, are disabled, or are physically and mentally impaired. ${ }^{40}$ Although this decreases the pressure on nursing homes and other long-term healthcare facilities, it increases pressure on both informal family caregivers and formal professional community-health caregivers. In the future, more and more older adults will be dependent, and fewer and fewer younger adults will be available to help them. ${ }^{41}$ Although there is some research and development on cognitive prosthetic devices, there are as yet few relevant tools, solutions, or technologies specifically for people with dementia. $^{42}$ To the best of our knowledge, there exist no clear overviews either of the research conducted by LLs in relation to cognitively impaired community-dwelling older adults, based on their observed and expressed needs, or of research using modern assistive technology specifically designed for community-dwelling older adults (with and without dementia).

Numerous studies have addressed areas of concern for ageing populations in general rather than specifically for those with dementia. ${ }^{4,44}$ Some have reported on the use of 
general memory aids, which can be used by those undergoing memory problems and other cognitive impairments. ${ }^{45}$ Mobile phone-based technologies have been reported as potential means of promoting social contact. ${ }^{46}$ Several solutions supporting ADL of community-dwelling older adults have been reported, including medication management services, item locators, and offering remote services for healthcare provision. ${ }^{47}$ However, these studies were often conducted in traditional laboratory settings and did not include older adults in their natural environments. Although such studies are easier to control, their ecological validity is compromised. ${ }^{48}$

Considering the needs of older adults with dementia in conjunction with relevant technologies, potentially innovative solutions for cognitive reinforcement have been identified. The increasing drive to develop innovative, costeffective dementia care strategies will only work effectively if innovative technologies meet the real needs of people living with dementia. These processes are often only discussed with their informal or professional caregivers, yet there is evidence that people with dementia are very capable of participating. ${ }^{49}$ Involving people with dementia in reallife context studies is challenging because of their impaired cognitive abilities, but studies, which do not include them will never be able to demonstrate the potential effects of implementation in real life. ${ }^{49}$ Within an LL, users play active roles in the design processes for innovations and drive the approach to innovation. ${ }^{28}$ Furthermore, LLs can involve people in their natural environments, thus providing more ecologically valid evaluations. ${ }^{48}$ Additionally, LLs focus on involving a network of relevant stakeholders. ${ }^{50}$ The value of exchanges in the context of innovations for dementia is based on the interactions between the stakeholders in the ecosystem. ${ }^{51}$ These stakeholders therefore need to have a role in the innovation process to ensure their needs are addressed. Our analysis of the literature identified five main themes. Firstly, scholars have attempted to conceptualize LLs and understand their positioning in the wider innovation literature. Secondly, studies pay attention to the virtual and physical environments of LLs in which multiple stakeholders contribute to innovation processes. Thirdly, many studies focus on stakeholder engagement in LLs. Although research acknowledges the presence of multiple stakeholders, the roles and engagement of users have emerged as the field's primary area of interest. Fourthly, the research discusses how co-creation should be managed in order to achieve the desired outcomes, and fifthly, studies highlight the challenges and successes of co-creation in LLs and describe the benefits that can be achieved by employing such an approach. Analyzing these themes in relation to summaries of each of the different needs of older adults with dementia is a particularly interesting aspect of this scoping review. A certain body of literature has already attempted to elucidate and analyze the effects of LLs on technology and communication ${ }^{50,52}$ However, each of the different and separate needs of older adults with dementia, and their respective solutions, remain under-researched and have yet to be summarized individually before presenting an detailed overall view of the state of the art. ${ }^{17}$

\section{Aims of the Scoping Review}

This protocol describes a scoping review of recent international reports and scientific publications on the services and research activities of LLs for and with older adults with dementia. The focus will be on those persons' needs and wants on the acronym PCC (participants, concepts, context). ${ }^{53}$ The following research question was developed to define our search of the available knowledge and publications: "What does the literature say about Living Labs whose activities are dedicated to older adults with dementia, whether they live in the community or in nursing homes?"

Specific objectives are:

- Identifying LL activities linked to older adults with dementia;

- Describing the fields of action of LLs dedicated to older adults with dementia and the types of research carried out;

- Investigating the technologies co-created in LLs to improve the autonomy and quality of life of older adults with dementia;

- Considering the impact of such solutions with regards to how effectively they reduce the encumber on informal and formal caregivers;

- Addressing how LLs have involved various stakeholders in identifying needs and finding solutions for older adults with dementia so that they can live more independently and with a better quality of life;

- What contributions do (geriatric) LLs make to the exploration of needs, testing technology, and applying a user-based approach with regards to community-dwelling older adults with dementia?

\section{Methods}

We will use the methodological framework for scoping reviews of Tricco et al (2018) published in the Equator 
network's website: The PRISMA recommendations for the Extension for Scoping Reviews (PRISMA-ScR) ${ }^{54}$ This framework suggests to include the research question, to identify and select relevant studies, and collecting, summarizing, and reporting the results of the retrieved studies. Two reviewers will independently review abstracts and full-text papers, with a consensus procedure in cases of disagreement. We will conduct a quality assessment of the selected papers using appropriate tools for bias analysis of quantitative, qualitative and mixed-method studies (Robins-E and the Pluye et al. -scoring system for appraising quantitative, qualitative and mixed-methods primary studies in Mixed Studies Reviews). ${ }^{55,56}$

\section{Eligibility Criteria}

Scoping review searches are recommended to be as comprehensive as possible in order to identify every possible study and reports on LL research activities/co-creation with older adults with dementia. ${ }^{57}$ Studies should provide a description of the co-creation process; research method/ design; the stakeholders involved; the impact/effect on autonomy/quality of life; or the impact/effect on health status, as defined by the authors.

Studies should be conducted within LLs or by researchers and managers (healthcare professionals, ICT experts, engineers) attached to an LL and working with older adults with dementia either living in the community or in nursing homes.

\section{Search Strategy}

We will conduct a systematic scoping review of published articles (quantitative, qualitative, and mixed-methods studies) in the following databases without date or language restrictions: Embase.com, Medline Ovid SP, Pubmed (NOT medline [sb]), PsychINFO Ovid SP, and Web of Science. We will also conduct supplementary searches by backwards citation searching, grey literature searches, and we plan to contact experts.

If studies are identified in languages other than those mastered by the research team, we will contact their authors and ask them to complete the data extraction and quality assessment forms. The database searches will be completed in March 2020. Retrieved articles will be screened, and the entire study is expected to be completed by December 2020. Table 1 presents the strings of the search strategy for the Embase.com database.

\section{Data Management}

Endnote ${ }^{\circledR} 9.2^{58}$ and Rayyan ${ }^{\circledR 59}$ will be used for collecting and analyzing the bibliographic references.

\section{Article Selection Process}

Studies will be selected by two independent reviewers. Each abstract will be evaluated and each potentially relevant full article will be retrieved for potential inclusion. Furthermore, data will be selected and extracted. Should the two reviewers disagree, the other team members will contribute, resolving the disagreement by discussion or, if needed, a consensus will be reached after further discussions with the authors. Article selection will be based on the methodological framework for scoping studies recommended by Tricco et al. ${ }^{54}$

\section{Data Collection Process}

This process will use Excel sheets for data on the studies and interventions, as well as for quality assessment of the studies, developed by the research team. Disagreements will be resolved through discussion and consultation with the co-authors.

\section{Data Items}

The following information will be extracted from each relevant study included and put into an appropriate usable form: (1) study authors, year of publication, and country where the study was conducted; (2) study characteristics (including research questions, study setting and design, sample size, instruments used, duration of follow-up, and stakeholders involved); (3) participants' characteristics (including age, sex, health status, and place of living); and (4) types of outcome measures. ${ }^{60}$

\section{Outcomes and Prioritization}

The scoping review's primary outcomes will be the nature, number, and assessment of studies carried out with older adults with dementia done by or in collaboration with LLs. Secondary outcomes will be the documentation of the different types of LL, their objectives, the location of their interventions, and their typologies and methods of co-creation for technologies and services for older adults and other stakeholders. These findings will enable us to put together an overview of existing LLs and their cocreation, service, and research activities. Overall, the outcomes of this scoping review will provide useful insights into existing activities and identify any remaining gaps in the services and research carried out in LLs. ${ }^{54}$

\section{Data Synthesis}

We will summarize the results using a descriptive narrative synthesis and content analysis. ${ }^{61,62}$ All the data on the LLs, their study objectives, co-creations, subjects, and 
Table I Overview of the Search Strings to Be Applied in EMBASE.com

\begin{tabular}{|l|l|}
\hline $\begin{array}{l}\text { Descriptors/ } \\
\text { Keywords/ }\end{array}$ & Search Strings AND/OR \\
\hline Living lab & $\begin{array}{l}\text { "participatory research'/de OR "participatory action } \\
\text { research/de OR ("living lab*" OR co-creat* OR co- } \\
\text { design* OR co-research OR co-conception OR } \\
\text { "participatory design" OR (participatory NEXT/3 } \\
\text { research) OR (innovation NEXT/3 communit*) OR } \\
\text { "user innovation" OR "community pilot"): ab,ti, kw }\end{array}$ \\
\hline $\begin{array}{l}\text { AND } \\
\text { cognitive } \\
\text { impairment/ } \\
\text { mental } \\
\text { disorders }\end{array}$ & $\begin{array}{l}\text { 'cognitive defect'/exp OR amnesia'/exp OR } \\
\text { (dementia* OR "cognitive impair*" OR "cognitive } \\
\text { defect" OR "neurocognitive disorder*" OR } \\
\text { "cognition disorder*" OR "traumatic psychose*" OR } \\
\text { Korsakoff OR Huntington OR "Lewy Body"):ab,ti,kw }\end{array}$ \\
\hline
\end{tabular}

stakeholders will be summarized in a table. By summarizing and critically appraising every study, we will be able to identify gaps in current work and avenues for future research for the benefit of older adults with dementia.

\section{Confidence in Cumulative Findings and Risk of Bias}

We will assess the quality of eligible experimental, observational and mixed-methods studies by using appropriate tools: Grading of Recommendations Assessment, Development and Evaluation (GRADE) and the Mixed-Methods Appraisal Tool (MMAT). ${ }^{63,64}$ Qualitative studies will be assessed using the "Qualitative evidence synthesis findings. ${ }^{95,66}$ The risk of bias in retained studies will be assessed using the RoB 2 tool for bias assessment for randomized and ROBINS-I for non-randomized studies. ${ }^{67,68} \mathrm{We}$ will not exclude studies based on their quality assessment because we wish to provide a comprehensive overview of the available information and its extent. The quality of information will be assessed using the following items: risk of bias, inconsistency, imprecision, indirectness, publication bias, and the confidence effect. ${ }^{69}$ Using the tools mentioned above, these six domains will be rated on a four-point Likert scale as: (1) very low, (2) low, (3) moderate, or (4) high. ${ }^{69}$ The Robvis web app will be employed for visualizing risk-of-bias assessments plots. ${ }^{70}$ Any disagreements in the ratings will be resolved by discussion.

\section{Results}

This comprehensive scoping review will examine the research and service activities carried out by LLs with older adults with dementia in different living environments. It will provide valuable information to healthcare providers, LL managers, policymakers, and other stakeholders involved in LLs dedicated to older adults. This protocol's significant contribution lies in the experience and insights that it will gather by applying and reflecting on the activities of LLs with older adults with dementia. This comprehensive scoping review protocol allows structuring and conceptualizing emerging and innovative concepts and research methodologies of the Living Lab approach in health care research among community-dwelling older adults with cognitive impairment. Living lab methodology and stakeholders will be considered as well the bottom-up user-centred data collection to offer a user-focused solution.

There has been a lack of such studies to date because it is difficult and challenging to involve cognitively impaired older adults in research projects. End-users should remain the most important stakeholders in LL studies, and in this regard, insights will be gathered on how cognitively impaired older adults could be more involved in LL studies. By allowing older adults with dementia to experience co-creation in a well-defined environment and to influence a potential product's design, ease of use or acceptability, the other stakeholders should be better able to address their needs. This should stimulate other researchers to perform their studies for people with dementia in suitable environments as well. This potentially more difficult research path is rarely chosen, ${ }^{38}$ despite such studies showing clear advantages, such as a user-pulled development rather than techno-pushed one. ${ }^{71}$ Our retrospective investigation will clearly identify the research methods applied in LLs and the kind of studies which best promote the roles, which older adults with dementia can fulfil. If we take this further, we will eventually be able to develop more suitable and sustainable innovative interventions to address the societal challenges of dementia.

\section{Discussion and Conclusion}

This documentary research project will provide a picture of the state of the art of LL research for older adults with dementia and reveal the extent of and gaps in the services provided and research carried out for them in different living environments. It will, therefore, provide valuable information to nurse and general practitioners, policymakers, and other stakeholders.

\section{Abbreviation}

ENoLL, The European Network of Living Labs. 


\section{Author Contributions}

All the authors contributed to the development of this comprehensive scoping review protocol, the design and the drafting of the manuscript, approved the final version of the paper to be published, and agreed to be held accountable for all aspects of the work.

\section{Disclosure}

All authors declare no conflicts of interest.

\section{References}

1. Eurostat. People in the EU: Who are We and How Do We Live? Publications Office of the European Union; 2015.

2. Statistical Office F. Population data, 2018 Confédération Suisse; 2018. Available form: https://www.bfs.admin.ch/bfs/en/home/statis tics/population.gnpdetail.2019-0274.html. Accessed September 1, 2019.

3. Cavalli S. Vieillards à domicile, vieillards en pension: Une comparaison [Old people at home, old people in pensions: a comparison] Suisse, Lausanne Ed. Réalités sociales; 2002. French.

4. Höpflinger F, Bayer-Oglesby L, Zumbrunn A. Pflegebedürftigkeit und langzeitpflege im Alter: Aktualisierte Szenarien für di Schweiz. Berne: Observatoire Suisse de la Santé; 2011.

5. FSO (Federal Statistical Office). Statistical Data on Switzerland. FSO; December, 2018. Available from: https://www.bfs.admin.ch/ bfs/en/home.html.

6. Lapointe D, Guimont D. Open innovation practices adopted by private stakeholders: perspectives for living labs. Info. 2015;17 (4):67-80. doi:10.1108/info-01-2015-0003

7. Thoma-Lürken T, Lexis MAS, Bleijlevens MHC, Hamers JPH. Development and usability of a decision support app for nurses to facilitate aging in place of people with dementia. Appl Nurs Res. 2018;42:35-44. doi:10.1016/j.apnr.2018.04.008

8. Angelini L, Carrino S, Abou Khaled O, Riva-Mossman S, Mugellini E. Senior Living Lab: an ecological approach to foster social innovation in an ageing society. Future Internet. 2016;8(4):50. doi:10.3390/fi8040050

9. Bächle M, Daurer S, Judt A, Mettler T. Assistive technology for independent living with dementia: stylized facts and research gaps. Health Policy Technol. 2018;7(1):98-111. doi:10.1016/j.hlpt.2017.12.002

10. Bergvall-Kareborn B, Hoist M, Stahlbrost A Concept design with a living lab approach. Paper presented at: System Sciences, 2009. HICSS'09. 42nd Hawaii International Conference; Big Island, HI, USA, 2009.

11. Coignard P, Joseph PA, Busnel M, Fattal C, Picard R, Laffont I. Living labs health and autonomy: what place for users and clinicians? The example of the French APPROCHE association. Ann Phys Rehabil Med. 2015;58:e57-e58. doi:10.1016/j.rehab.2015.07.134

12. Living Labs EN. European Network of Living Labs; E-U, ed. 2019. Brussels:ENoLL. Available from: https://enoll.org/newsroom/. Accessed January 13, 2020.

13. Sauer SC. User Innovativeness in Living Laboratories: Everyday User Improvisations with ICTs as a Source of Innovation. Universiteit Twente; 2013.

14. Malmberg K, Vaittinen I. Living Lab Methodology: Handbook. Brussels: European Network of Living Labs; 2017.

15. Mitchell WJ. Me++: The Cyborg Self and the Networked City. Cambridge: MIT Press; 2003.

16. Schuurman D, De Moor K, De Marez L, Evens T. A Living Lab research approach for mobile TV. Telemat Inform. 2011;28 (4):271-282. doi:10.1016/j.tele.2010.11.004
17. Ballon P, Schuurman D. Living labs: concepts, tools and cases. Info. 2015;4:1-11.

18. Dutilleul B, Birrer FAJ, Mensink W. Unpacking european living labs: analysing innovation's social dimensions. Cent Eur J Public Policy. 2010;4(1):60-85.

19. Kviselius NZ. Living labs as tools for open innovation. Commun Strategies. 2009;2(74):75-94.

20. Tang T, Wu Z, Karhu K, Hämäläinen M, Ji Y. Internationally distributed living labs and digital ecosystems for fostering local innovations in everyday life. J Emerging Technol Web Intell. 2012;4 (1):106-115. doi:10.4304/jetwi.4.1.106-115

21. Grotenhuis FDJ. Living labs as service providers: from proliferation to coordination. Global Bus Organ Excellence. 2017;36(4):52-57. doi: $10.1002 /$ joe. 21790

22. Mulder I, Velthausz D, Kriens M. The living labs harmonization cube: communicating living lab's essentials. Electron J Virtual Organ Netw. 2008;10:1-14.

23. Bergvall-Kåreborn B, Ståhlbröst A. Living lab: an open and citizen-centric approach for innovation. Int J Innovation Reg Dev. 2009;1(4):356-370. doi:10.1504/IJIRD.2009.022727

24. Leminen S, Nystrom AG, Westerlund M. A typology of creative consumers in living labs. J Eng Technol Manage. 2015;37:6-20. doi:10.1016/j.jengtecman.2015.08.008

25. Schuurman D. Bridging the Gap Between Open and User Innovation?: Exploring the Value of Living Labs as a Means to Structure User Contribution and Manage Distributed Innovation. Ghent: Department of Communication Sciences, University of Ghent; 2015.

26. Bergvall-Kåreborn B, Eriksson CI, Ståhlbröst A, Svensson J. A milieu for innovation: defining living labs. ISPIM Innovation Symposium; 6 December 2009. New York, NY: USA; 2009.

27. Schaffers H, Turkama P. Living labs for cross-border systemic innovation. Technol Innovation Manage Rev. 2012;2:25-30. doi: $10.22215 /$ timreview/605

28. Almirall E. Mapping living labs in the landscape of innovation methodologies. Technol Innovation Manage Rev. 2012;2(9):12-18. doi:10.22215/timreview/603

29. Følstad A. Living Labs for innovation and development of information and communication technology: a literature review. Electron $J$ Virtual Organ Netw. 2008;10:7.

30. Westerlund M, Leminen S. Managing the challenges of becoming an open innovation company: experiences from living labs. Technol Innovation Manage Rev. 2011;10:19-25. doi:10.22215/timreview/489

31. Payne A, Storbacka K, Frow P, Knox S. Co-creating brands: diagnosing and designing the relationship experience. J Bus Res. 2009;62 (3):379-389. doi:10.1016/j.jbusres.2008.05.013

32. Almirall E, Wareham J. Living labs and open innovation: roles and applicability. Electron J Virtual Organ Netw. 2008;10(3):21-46.

33. Cleland B. Innovation of eParticipation strategies using living labs as intermediaries. Electron J e-Government. 2012;10(2):120-132.

34. Lethin C, Rahm Hallberg I, Renom Guiteras A, et al. Prevalence of dementia diagnoses not otherwise specified in eight European countries: a cross-sectional cohort study. BMC Geriatr. 2019;19(1):172. doi:10.1186/s12877-019-1174-3

35. Fischler I. Using technology to implement the health quality Ontario quality standards for behavioural symptoms of dementia. Alzheimer's Dementia. 2017;13(7):P1558. doi:10.1016/j.jalz.2017.07.705

36. Kales HC, Gitlin LN, Lyketsos CG. Assessment and management of behavioral and psychological symptoms of dementia. BMJ. 2015;350:h369. doi:10.1136/bmj.h369

37. Gray LC, Bernabei R, Berg K, et al. Standardizing assessment of elderly people in acute care: the interRAI acute care instrument. $J \mathrm{Am}$ Geriatr Soc. 2008;56(3):536-541. doi:10.1111/(ISSN)1532-5415

38. Topo P. Technology studies to meet the needs of people with dementia and their caregivers: a literature review. J Appl Gerontol. 2009;28 (1):5-37. doi:10.1177/0733464808324019 
39. Cohen-Mansfield J, Werner P, Reisberg B. Temporal order of cognitive and functional loss in a nursing home population. $J$ Am Geriatr Soc. 1995;43(9):974-978. doi:10.1111/jgs.1995.43.issue-9

40. Boldy D, Grenade L, Lewin G, Karol E, Burton E. Older people's decisions regarding 'ageing in place': a Western Australian case study. Australas J Ageing. 2011;30(3):136-142. doi:10.1111/ ajag.2011.30.issue-3

41. North MS, Fiske ST. Modern attitudes toward older adults in the aging world: a cross-cultural meta-analysis. Psychol Bull. 2015;141 (5):993-1021. doi:10.1037/a0039469

42. Petonito G, Muschert GW. Silver alert: societal aging, dementia, and framing a social problem. In: Critical Gerontology Comes of Age. Routledge: Taylor \& Francis Group; 2018:146-162.

43. Bourazeri A, Stumpf S Co-designing smart home technology with people with dementia or Parkinson's disease. Proceedings of the 10th Nordic Conference on Human-Computer Interaction; 2018; Oslo, Norway.

44. Meiland F, Innes A, Mountain G, et al. Technologies to support community-dwelling persons with dementia: a position paper on issues regarding development, usability, effectiveness and cost-effectiveness, deployment, and ethics. JMIR Rehabil Assist Technol. 2017;4(1):e1. doi:10.2196/rehab.6376

45. Van der Roest HG, Wenborn J, Pastink C, Droes RM, Orrell M. Assistive technology for memory support in dementia. Cochrane Database Syst Rev. 2017;6:Cd009627.

46. Mehra S, Visser B, Cila N, et al. Supporting older adults in exercising with a tablet: a usability study. JMIR Hum Factors. 2019;6(1): e11598. doi:10.2196/11598

47. Gibson G, Brittain K, Robinson L. Working with assistive technologies and people living with dementia. In: Neves BB, Vetere F, editors. Ageing and Digital Technology: Designing and Evaluating Emerging Technologies for Older Adults. Singapore: Springer Singapore; 2019:213-227.

48. Koskinen I, Zimmerman J, Binder T, Redström J, Wensveen S. Design Research Through Practice from the Lab, Field, and Showroom. Waltham, MA: Morgan Kaufmann; 2011.

49. Astell AJ, Ellis MP, Bernardi L, et al. Using a touch screen computer to support relationships between people with dementia and caregivers. Interact Comput. 2010;22(4):267-275. doi:10.1016/j. intcom.2010.03.003

50. Leminen S, Westerlund $\mathrm{M}$, Nyström AG. Living labs as open-innovation networks. Technol Innovation Manage Rev. 2012;2:6-11. doi:10.22215/timreview/602

51. Payne A, Ballantyne D, Christopher M. A stakeholder approach to relationship marketing strategy the development and use of the "six markets" model. Eur J Mark. 2005;39(7-6):855-871. doi:10.1108/ 03090560510601806

52. Følstad A. Living Labs for innovation and development of information and communication technology: a literature review. Electron J Virtual Organ Netw. 2008;10(8):99-131.

53. Lockwood C, dos Santos KB, Pap R. Practical Guidance for Knowledge Synthesis: Scoping Review Methods. Asian Nursing Research. 2019;13(5):287-294

54. Tricco AC, Lillie E, Zarin W, et al. PRISMA extension for scoping reviews (PRISMA-ScR): checklist and explanation. Ann Intern Med. 2018;169(7):467-473. doi:10.7326/M18-0850

55. Pluye P, Gagnon MP, Griffiths F, Johnson-Lafleur J. A scoring system for appraising mixed methods research, and concomitantly appraising qualitative, quantitative and mixed methods primary studies in mixed studies reviews. Int J Nurs Stud. 2009;46(4):529-546. doi:10.1016/j. ijnurstu.2009.01.009
56. Morgan R, Sterne J, Higgins J, Thayer K, Schunemann H, Rooney A. A new instrument to assess risk of bias in non-randomised studies of exposures (ROBINS-E): application to studies of environmental exposure. Cochrane Database Syst Rev. 2017;9.

57. Munn Z, Peters MDJ, Stern C, Tufanaru C, McArthur A, Aromataris E. Systematic review or scoping review? Guidance for authors when choosing between a systematic or scoping review approach. BMC Med Res Methodol. 2018;18(1):143. doi:10.1186/s12874-018-0611-x

58. Endnote [Computer Program]. Version 9.2. Philadelphia: Web of Science Group; 2019.

59. Ouzzani M, Hammady H, Fedorowicz Z, Elmagarmid A. Rayyan-a web and mobile app for systematic reviews. Syst Rev. 2016;5(1):210. doi:10.1186/s13643-016-0384-4

60. Aguayo-Albasini JL, Flores-Pastor B, Soria-Aledo V. Sistema GRADE: clasificación de la calidad de la evidencia y graduación de la fuerza de la recomendación [GRADE system: classification of quality of evidence and strength of recommendation]. Cirugea Espanola. 2014;92(2):82-88. doi:10.1016/j.ciresp.2013.08.002 Spanish.

61. Souto RQ, Khanassov V, Hong QN, Bush PL, Vedel I, Pluye P. Systematic mixed studies reviews: updating results on the reliability and efficiency of the mixed methods appraisal tool. Int J Nurs Stud. 2015;52(1):500-501. doi:10.1016/j.ijnurstu.2014.08.010

62. Dixon-Woods M, Agarwal S, Jones D, Young B, Sutton A. Synthesising qualitative and quantitative evidence: a review of possible methods. J Health Serv Res Policy. 2005;10(1):45-53. doi:10. $1177 / 135581960501000110$

63. Guyatt GH, Oxman AD, Sultan S, et al. GRADE guidelines: 9. Rating up the quality of evidence. J Clin Epidemiol. 2011;64 (12):1311-1316. doi:10.1016/j.jclinepi.2011.06.004

64. Pace R, Pluye P, Bartlett G, et al. Testing the reliability and efficiency of the pilot Mixed Methods Appraisal Tool (MMAT) for systematic mixed studies review. Int $J$ Nurs Stud. 2012;49(1):47-53. doi:10.1016/j.ijnurstu.2011.07.002

65. Zeng X, Zhang Y, Kwong JS, et al. The methodological quality assessment tools for preclinical and clinical studies, systematic review and meta-analysis, and clinical practice guideline: a systematic review. J Evid Based Med. 2015;8(1):2-10. doi:10.1111/jebm.12141

66. Glenton C, Carlsen B, Lewin S, et al. Applying GRADE-CERQual to qualitative evidence synthesis findings-paper 5: how to assess adequacy of data. Implement Sci. 2018;13(Suppl 1):14. doi:10.1186/ s13012-017-0692-7

67. Sterne JA, Hernán MA, Reeves BC, et al. ROBINS-I: a tool for assessing risk of bias in non-randomised studies of interventions. BMJ. 2016;355:i4919. doi:10.1136/bmj.i4919

68. Sterne JAC, Savovic J, Page MJ, et al. RoB 2: a revised tool for assessing risk of bias in randomised trials. BMJ. 2019;366:14898. doi: $10.1136 / \mathrm{bmj} .14898$

69. Shea BJ, Reeves BC, Wells G, et al. AMSTAR 2: a critical appraisal tool for systematic reviews that include randomised or non-randomised studies of healthcare interventions, or both. $B M J$. 2017;358:j4008. doi:10.1136/bmj.j4008

70. McGuinness LA. Robvis: an R package and web application for visualising risk-of-bias assessments; 2019. Available from: https: www.eshacktron.org/software/robvis.html. 2019.

71. Bharucha AJ, Anand V, Forlizzi J, et al. Intelligent assistive technology applications to dementia care: current capabilities, limitations, and future challenges. Am J Geriatr Psychiatry. 2009;17(2):88-104. doi:10.1097/JGP.0b013e318187dde5 


\section{Publish your work in this journal}

Smart Homecare Technology and TeleHealth is an international, peer-reviewed, open access online journal publishing original research, reviews, editorials and commentaries on the application of technology to support people and patients at home and in assisted living centers to optimize healthcare and management resources. Specific topics in the journal include: Development and application of devices within the home and embedded in appliances; Healthcare provider communication and education tools; and drug ordering and adherence. The manuscript management system is completely online and includes a very quick and fair peer-review system, which is all easy to use. Visit http://www.dovepress.com/testimonials.php to read real quotes from published authors. 